\title{
COMBINATORIAL METHODS FOR THE TWISTED COHOMOLOGY OF ARTIN GROUPS
}

\author{
M. SAlvetti AND A. Villa
}

\begin{abstract}
In this paper we introduce certain "combinatorial sheaves" on posets, which we call weighted sheaves, and we relate their cohomology, computed by a weighted complex, with the twisted cohomology of Artin groups. It turns out that to each Artin group one can associate a weighted sheaf, where the poset is given by the simplicial complex of all finite parabolic subgroups, and the cohomology of the Artin group with coefficients in a module of Laurent polynomials (interesting for geometrical reasons) is computed by the associated weighted complex. We connect this theory with the so called "Discrete Morse Theory": a weighted matching on the weighted complex gives rise to a Morse complex computing the cohomology. We give a natural filtration of the weighted complex, which is compatible with the weighted matching, so obtaining a converging spectral sequence. We use such machinery to compute the twisted conomology for all exceptional type affine Artin groups.
\end{abstract}

\section{Introduction}

Let $(\mathbf{W}, S)$ be a (finitely generated) Coxeter system and let $\mathbf{G}_{\mathbf{W}}$ be its associated Artin group. For example, one can take as $\mathbf{W}$ the symmetric group $\Sigma_{n}$ with set of Coxeter generators the transpositions $(i, i+1)$, and as $\mathbf{G}_{\mathbf{W}}$ the braid group $\mathrm{Br}_{n}$. There is a naturally associated configuration space $\mathbf{Y}$ where $\mathbf{W}$ acts freely, which is conjectured to be a $K(\pi, 1)$-space; such conjecture is verified for some (few) cases, including the case when $\mathbf{W}$ is a finite group. In all cases, the topology of the orbit space $\mathbf{Y}_{\mathbf{W}}:=\mathbf{Y} / \mathbf{W}$ is interesting to study. Its cellular description is given in [23] (see also $[6-8,21])$, where a $\mathbf{C W}$-complex $\mathbf{X}_{\mathbf{W}}$ which is a deformation retract is explicitly described.

We are interested in a natural representation of the Artin group over the ring of Laurent polynomials in one variable $q$, taking each standard generator to the $q$ multiplication. For finite-type Artin groups and Laurent polynomials over $\mathbb{Q}$, the cohomology with coefficients in such representation is fully computed (see $[10,11,16]$, [9]; see [5] for Laurent polynomials over $\mathbb{Z}$ ). For affine-type Artin groups some cases are worked out (see [6-8]).

In this paper, we apply a new combinatorial procedure (introduced by the first author and partially described also in $[20,21,24])$ to calculate the twisted cohomology of the orbit space.

The main combinatorial ingredient is the introduction of a very special kind of sheaves over posets, which we call weighted sheaves over posets, which are interesting objects in themselves. The most important case for us is when such poset is the face poset of a simplicial complex $K$. We define a weighted sheaf as a triple $(K, R, w)$

Received by the editors March 29, 2013. 
where $R$ is a ring (usually a Principal Ideal Domain, PID for brevity) and $w$ is a map between the face poset of $K$ and the ring $R$, which respects the inclusion relation in $K$ and the divisibility relation in $R: \sigma \subset \tau \Rightarrow w(\sigma) \mid w(\tau)$. To these data one associates an algebraic complex $L_{*}$, which we call here the weighted complex, which is a torsion complex generated in dimension $n$ as $L_{n}:=\bigoplus_{|\sigma|=n} \frac{R}{(w(\sigma))} \bar{e}_{\sigma}$. The boundary is induced by the boundary in $K$ and by the inclusion maps.

A weighted sheaf $(K, R, w)$ is naturally associated to an Artin group. Here $K$ is the simplicial complex, defined over $S$, of all subsets $J \subset S$ such that the parabolic subgroup $\mathbf{W}_{J}$ is finite; $R$ is the ring of Laurent polynomials, and $w(J)$ is the Poincaré polynomial of $\mathbf{W}_{J}$. From the homology of the weighted complex, one easily derives that of the orbit space, since the two are related in a long exact sequence, where (for affine type Artin groups) there are isomorphisms except in the top dimension.

One can localize the weighted complex at the irreducible factors of the Poincaré polynomials (which turn out to be cyclotomic polynomials); there is a natural filtration of the localized complexes, and therefore an associated spectral sequence whose $E^{1}$ term is computed as relative (untwisted) homology of simplicial subcomplexes of $K$ (Theorem 1).

The second combinatorial ingredient is Discrete Morse Theory, which was introduced in $[14,15]$ (see also [19]). We use a small variation of the standard theory, by calling weighted matching on a weighted complex a matching $\mathcal{M}$ in the standard sense on the poset of the faces in $K$, together with the additional condition that two matched elements must have the same weight. Such matching gives rise to an associated Morse complex $C_{*}^{\mathcal{M}}$ : as a module, $C_{*}^{\mathcal{M}}$ is the torsion complex generated by the critical cells; the boundary is given by "combinatorial integration" of the flow over alternating paths.

We show that such notions fit very well into the theory of weighted sheaves. First, we prove that the Morse complex computes the (co-)homology of the weighted complex, so generalizing the known results for free modules (Theorem 2).

Second, the above filtration of the weighted complex induces a filtration onto the Morse complex and an isomorphism of spectral sequences (Theorems 3 and 4).

To apply the previous theory and perform computations, we take a suitable weighted matching on the poset of subgraphs of the Coxeter diagram of the group. We describe some natural ways to take such matchings, and we give in details the example of the affine case $\widetilde{\mathrm{F}}_{4}$.

We succeed to make complete calculations (by hand) for all the exceptional affinetype Artin groups. We include at the end the tables containing all the homology groups and their generators.

\section{Weighted sheaves over posets}

Let $(P, \prec)$ be a poset.

Definition 2.1. A sheaf of rings over $P$ (also called diagram of rings) is a collection

$$
\left\{R_{x}, x \in P\right\}
$$

of commutative rings together with a collection of ring homomorphisms

$$
\left\{\rho_{x, y}: R_{y} \rightarrow R_{x}, \quad x \preceq y\right\}
$$


satisfying

$$
\begin{gathered}
\rho_{x, x}=i d_{R_{x}} ; \\
x \preceq y \preceq z \Rightarrow \rho_{x, z}=\rho_{x, y} \rho_{y, z}
\end{gathered}
$$

(see also $[1,26])$.

It is convenient to consider $P$ as a category with objects all elements of $P$, and a unique morphism between $x$ and $y$ if $x \preceq y$ (or no morphism otherwise). So, one can see a sheaf of rings as a contravariant functor from $P$ as a category to the category of rings.

We take now a unique factorization domain $R$ (later we assume that $R$ is a PID).

We consider in $R$ the transitive relation given by divisibility in $R$ : we write $x \mid y$ when $\exists z \in R$ such that $y=x z$. Analog to $P$ and $\preceq$, the relation of divisibility gives $R$ the structure of a small category (of course, such relation induces a structure of poset on the set $R / R_{*}$, where $R_{*}$ is the multiplicative subgroup of units in $R$ ).

In particular, any morphism

$$
w:(P, \prec) \rightarrow(R, \mid): x \longrightarrow w(x):=w_{x} \in R
$$

defines a sheaf over $P$ by the collections

$$
\left\{R /\left(w_{x}\right), x \in P\right\}
$$

and

$$
\left\{i_{x, y}: R /\left(w_{y}\right) \rightarrow R /\left(w_{x}\right)\right\}
$$

where $i_{x, y}$ is the map induced by the identity of $R$, well defined since by definition $\left(w_{y}\right) \subset\left(w_{x}\right)$.

The following is the main definition of this paper.

Definition 2.2. We call the triple $(P, R, w)$ a weighted sheaf over $P$ and the coefficients $w_{x}$ the weights of the sheaf.

Example 1. When the poset is given by a simplicial complex (or a simplicial scheme) $K$ with partial ordering

$$
\sigma \prec \tau \Leftrightarrow \sigma \subset \tau
$$

then a weighted sheaf over $K$ is given by assigning to each simplex $\sigma \in K$ a weight $w_{\sigma} \in R$, with

$$
\sigma \prec \tau \Rightarrow w_{\sigma} \mid w_{\tau} .
$$

Remark 2.3. Recall that the order complex $\Delta(P)$ of a poset $P$ is the simplicial complex with vertex set $P$ and $q$-simplices the $q+1$-chains of $P$.

A weighted sheaf $(P, R, w)$ over a poset $P$ induces a weighted sheaf $\left(\Delta(P), R, w^{\prime}\right)$ over $\Delta(P)$. It is sufficient to define the weight $w^{\prime}$ of a chain $c$ of $P$ as

$$
w^{\prime}(c):=w(\max (c)) .
$$

Remark 2.4. The previous definition 2.2 (and part of the following theory) could be extended to the more general setting where one associates to any $x \in P$ an ideal $I_{x}$ in some fixed $\operatorname{ring} A$, with the condition $x \prec y \Rightarrow I_{y} \subset I_{x}$. Our setting corresponds to the case, where $I_{x}$ is a principal ideal, for all $x \in P$. 
In the following, we consider mainly the case when the poset is a simplicial scheme $K$ (see Example 1), defined over some finite set $S$.

Let $C_{*}^{0}(K)$ be the algebraic complex for the reduced simplicial homology of $K$ with coefficients in $R$, shifted in dimension by one. In dimension $k$ one has

$$
C_{k}^{0}(K)=\bigoplus_{\substack{\sigma \in K,|\sigma|=k}} R \cdot e_{\sigma}^{0}
$$

where $e_{\sigma}^{0}$ is a generator associated to a given orientation of $\sigma$. In dimension 0 by definition, we have $C_{0}^{0}(K)=R e_{\emptyset}^{0}$.

The boundary is given by

$$
\partial^{0}\left(e_{\sigma}^{0}\right)=\sum_{|\tau|=k-1}[\tau: \sigma] e_{\tau}^{0},
$$

where $[\tau: \sigma]$ denotes the incidence number holding \pm 1 if $\tau \prec \sigma$ and vanishing otherwise.

The second main definition concerns a modified complex for the homology of weighted sheaves over a simplicial scheme $K$.

Definition 2.5. The weighted complex associated to the weighted sheaf $(K, R, w)$ is the algebraic complex

$$
L_{*}:=L_{*}(K)
$$

defined by

$$
L_{k}:=\bigoplus_{|\sigma|=k} \frac{R}{\left(w_{\sigma}\right)} \bar{e}_{\sigma}, \quad k \geq 0
$$

and boundary

$$
\partial: L_{k} \rightarrow L_{k-1}
$$

induced by $\partial^{0}$ :

$$
\partial\left(a_{\sigma} \bar{e}_{\sigma}\right)=\sum_{\tau \prec \sigma}[\tau: \sigma] i_{\tau, \sigma}\left(a_{\sigma}\right) \bar{e}_{\tau}
$$

The condition $\partial^{2}=0$ is straightforward from the definitions.

We will be interested to the homology $H_{*}\left(L_{*}\right)$, which we will refer to as the homology of the weighted sheaf $(K, R, w)$.

Remark 2.6. If $(P, R, w)$ is a weighted sheaf over a poset $P$ and $\left(K=\Delta(P), R, w^{\prime}\right)$ is the induced weighted sheaf as in Remark 2.3 then the associated weighted complex

$$
L_{*}(K)=\bigoplus_{x_{1} \prec \cdots \prec x_{k}} \frac{R}{\left(w\left(x_{k}\right)\right)} \bar{e}\left(x_{1} \prec \cdots \prec x_{k}\right)
$$

has boundary

$$
\partial\left(a \bar{e}\left(x_{1} \prec \cdots \prec x_{k}\right)\right)=\sum_{m=1}^{k}(-1)^{m} a^{\prime} \bar{e}\left(x_{1} \prec \cdots \prec \widehat{x_{m}} \prec \cdots \prec x_{k}\right),
$$

where $a^{\prime}:=a$ if $m<k$, while $a^{\prime}=i_{x_{k-1}, x_{k}}(a)$ if $m=k$. 
2.0.1. Decomposition and filtration of $L_{*}(K)$. We assume hereafter that $R$ is a PID.

Let $L:=L_{*}(K)$ be the algebraic complex associated to $(K, R, w)$, where $K$ is as in Example 1.

Each single factor $\frac{R}{\left(w_{\sigma}\right)}$ of $L$ decomposes into cyclic modules according to the irreducible factorization of $w_{\sigma}$ :

$$
w_{\sigma}=\prod_{i} \varphi_{i}^{m_{i}} \Rightarrow \frac{R}{\left(w_{\sigma}\right)} \cong \bigoplus_{i} \frac{R}{\left(\varphi_{i}^{m_{i}}\right)} .
$$

Given any irreducible $\varphi \in R$, we define the $\varphi$-primary component of the weighted sheaf through the $\varphi$-adic valuation as follows. For $\sigma \in K$, let

$$
v_{\varphi}(\sigma):=\max \text { power of } \varphi \text { dividing } w_{\sigma}
$$

and let $w_{(\varphi)}: K \rightarrow R$ be defined by

$$
w_{(\varphi)}(\sigma):=\varphi^{v_{\varphi}(\sigma)} .
$$

Then $\left(K, R, w_{(\varphi)}\right)$ defines a weighted sheaf, which we call the $\varphi$-primary component of the given sheaf, and denote also by $(K, R, w)_{(\varphi)}$. The associated complex is $L_{(\varphi)}$, defined in degree $k$ by:

$$
\left(L_{(\varphi)}\right)_{k}:=\bigoplus_{|\sigma|=k} \frac{R}{\left(\varphi^{v_{\varphi}(\sigma)}\right)} \bar{e}_{\sigma}
$$

There is a natural increasing filtration into subcomplexes of $L_{(\varphi)}$ by the powers of $\varphi$ :

$$
F^{s}\left(L_{(\varphi)}\right):=\bigoplus_{v_{\varphi}(\sigma) \leq s} \frac{R}{\left(\varphi^{v_{\varphi}(\sigma)}\right)} \bar{e}_{\sigma} .
$$

The latter is associated to an increasing filtration of the simplicial complex $K$ into subcomplexes:

$$
K_{(\varphi), s}:=\left\{\sigma \in K \mid v_{\varphi}(\sigma) \leq s\right\} .
$$

Then $F^{s}\left(L_{(\varphi)}\right)$ is the weighted complex associated to the weighted sheaf $\left(K_{(\varphi), s}, R\right.$, $\left.w_{(\varphi)} \mid K_{(\varphi), s}\right)$.

We get the following theorem.

Theorem 1. Let $(K, R, w)$ be a weighted sheaf, with associated weighted complex $L_{*}$. For any irreducible $\varphi \in R$, there exists a spectral sequence

$$
E_{p, q}^{0} \quad \Rightarrow \quad H_{*}\left(L_{(\varphi)}\right)
$$

that abuts to the homology of the $\varphi$-primary component of the associated algebraic complex $L_{*}$.

Moreover the $E^{1}$-term:

$$
E_{p, q}^{1}=H_{p+q}\left(F^{p} / F^{p-1}\right) \cong H_{p+q}\left(K_{(\varphi), p}, K_{(\varphi), p-1} ; R /\left(\varphi^{p}\right)\right)
$$

is isomorphic to the relative homology with trivial coefficients of the simplicial complexes pair $\left(K_{(\varphi), p}, K_{(\varphi), p-1}\right)$. 
Proof. The only non-standard fact is the second isomorphism in (2.6), with a relative homology group with trivial coefficients. This follows straightforward since by definition the boundary $\partial$ of $L_{(\varphi)}$ restricted to $F^{p} L_{(\varphi)} / F^{p-1} L_{(\varphi)}$ reduces to the standard boundary $\partial^{0} \otimes i d$ of $C_{*}^{0} \otimes \frac{R}{\left(\varphi^{p}\right)}$.

\section{Discrete Morse theory for weighted sheaves}

We show here how the discrete Morse theory for complexes generalizes to weighted sheaves (we refer in this section to $[14,15,19]$ ).

Given $x, y$ in a poset $P$, the notation $x \triangleleft y$ means that $y$ covers $x$, that is $x \prec y$ and there is no other $z \in P$ such that $x \prec z \prec y$.

Recall that a matching in a poset $P$ is defined as a set $\mathcal{M} \subset P \times P$ such that $(x, y) \in \mathcal{M} \Rightarrow x \triangleleft y$ and each $x \in P$ belongs to at most one pair of $\mathcal{M}$.

An alternating path is a sequence

$$
y_{0} \triangleright x_{1} \triangleleft y_{1} \triangleright x_{2} \triangleleft y_{2} \triangleright \cdots \triangleright x_{m} \triangleleft y_{m}\left(\triangleright x_{m+1}\right)
$$

such that each pair $x_{i} \triangleleft y_{i}$ belongs to $\mathcal{M}$ and no pair $x_{i} \triangleleft y_{i-1}$ belongs to $\mathcal{M}$.

A cycle is a closed alternating path with $y_{0}=y_{m}$.

An acyclic matching over $P$ is a matching with no cycles.

(The original definition of discrete vector field over the cells of a $C W$-complex, see [14], translates into the above notion of matching in the associated face-poset, see for example [19]). An acyclic matching can be associated to a discrete Morse function [14].

Definition 3.1. A weighted acyclic matching on a weighted sheaf $(P, R, w)$ over $P$ is an acyclic matching $\mathcal{M}$ on $P$ such that

$$
x \triangleleft y \in \mathcal{M} \Rightarrow w(x)=w(y)
$$

(We will refer briefly just to an acyclic matching on the weighted sheaf, if it is clear from the context).

Remark 3.2. In the more general situation of Remark 2.4, one could define a weighted acyclic matching on a weighted sheaf $(P, R, w)$ as an acyclic matching $\mathcal{M}$ such that $x \triangleleft y \in \mathcal{M}$ implies that the ideal $I_{x}$ equals the ideal $I_{y}$.

Let now $\mathcal{S}:=(K, R, w)$ be a weighted sheaf as in the previous section, where $K$ is a simplicial complex with natural partial ordering (2.1). Given an irreducible $\varphi$, let $\mathcal{S}_{(\varphi)}:=\left(K, R, w_{(\varphi)}\right)$ be the $\varphi$-primary component defined in the previous section, with associated weighted complex $L_{(\varphi)}($ see $(2.4))$.

Let $\mathcal{M}$ be a weighted acyclic matching for $\mathcal{S}_{(\varphi)}$. The standard theory generalizes as follows.

Definition 3.3. (1) A critical simplex of $K$ is a simplex $\sigma$ which does not belong to any pair of $\mathcal{M}$.

(2) The Morse complex of $\mathcal{S}$, with respect to $\mathcal{M}$, is defined as the (torsion) complex

with boundary

$$
C_{*}^{\mathcal{M}}:=\bigoplus_{\sigma \text { critical }} \frac{R}{\left(w_{\sigma}\right)} \bar{e}_{\sigma}
$$




$$
\partial^{\mathcal{M}}\left(a \bar{e}_{\sigma}\right):=\sum_{\substack{\tau \text { critical } \\|\tau|=|\sigma|-1}}\left(\sum_{p} \iota_{p, \tau, \sigma}(a)\right) \bar{e}_{\tau}
$$

Here the sum is over all alternating paths starting with $\sigma$ and ending with $\tau$. Such path

$$
p=\sigma \triangleright \tau_{1} \triangleleft \sigma_{1} \triangleright \tau_{2} \triangleleft \sigma_{2} \triangleright \cdots \triangleright \tau_{m} \triangleleft \sigma_{m} \triangleright \tau
$$

contributes by

$$
\begin{aligned}
& \iota_{p, \tau, \sigma}(a):=(-1)^{m}\left(\left[\tau: \sigma_{m}\right] i_{\tau, \sigma_{m}}\right) \circ\left(\left[\tau_{m}: \sigma_{m}\right]^{-1} i_{\tau_{m}, \sigma_{m}}^{-1}\right) \circ \cdots \\
& \ldots \circ\left(\left[\tau_{2}: \sigma_{1}\right] i_{\tau_{2}, \sigma_{1}}\right) \circ\left(\left[\tau_{1}: \sigma_{1}\right]^{-1} i_{\tau_{1}, \sigma_{1}}^{-1}\right) \circ\left(\left[\tau_{1}: \sigma\right] i_{\tau_{1}, \sigma}\right)(a),
\end{aligned}
$$

where the inverse of each $i_{\tau_{j}, \sigma_{j}}$ is well defined: by definition of weighted matching, $w_{\tau_{j}}=w_{\sigma_{j}}$, so $i_{\tau_{j}, \sigma_{j}}$ is actually the identity map.

So we can rewrite (3.3) in a more compact way as

$$
\iota_{p, \tau, \sigma}(a):=(-1)^{m}\left[\tau: \sigma_{m}\right] i_{\tau, \sigma_{m}} \circ\left[\prod_{j=m-1}^{0} \frac{\left[\tau_{j+1}: \sigma_{j}\right]}{\left[\tau_{j+1}: \sigma_{j+1}\right]} i_{\tau_{j+1}, \sigma_{j+1}}^{-1} \circ i_{\tau_{j+1}, \sigma_{j}}\right](a),
$$

where we set $\sigma_{0}=\sigma$.

Next, we generalize some standard results in "algebraic Morse theory" to our context.

Let $A$ be a PID and let $D$ be an $A$-module. For $d \in D$, we define the torsion of $d$ as the principal ideal

$$
\operatorname{tors}(d):=\{a \in A: a \cdot d=0\} .
$$

We need the following property of PID's.

Proposition 3.4. Let $A$ be a PID and let $D$ be an A-module with generators $f_{1}, \ldots$, $f_{k}$, and a direct sum decomposition into cyclic submodules

$$
D=\oplus_{i=1}^{k} A \cdot f_{i},
$$

where $\operatorname{tors}\left(f_{i}\right)=\left(d_{i}\right)$, so $A . f_{i} \cong \frac{A}{\left(d_{i}\right)}, i=1, \ldots, k$.

Let

$$
\left\{\begin{array}{l}
g_{1}=f_{1}, \\
g_{2}= \pm f_{2}+\sum_{j \geq 3} c_{j 2} f_{j}, \\
g_{i}=f_{i}+c_{1 i} f_{1}, \quad i=3, \ldots, k
\end{array}\right.
$$

be another set of generators of $D$, with same torsion

$$
\operatorname{tors}\left(g_{i}\right)=\operatorname{tors}\left(f_{i}\right), \quad i=1, \ldots, k .
$$

Then we have a decomposition

$$
D=\oplus_{i=1}^{k} A \cdot g_{i} .
$$


Proof. We need to proof that

$$
\sum_{i=1}^{k} a_{i} g_{i}=0, a_{i} \in A, i=1, \ldots, k \Rightarrow a_{i} \in \operatorname{tors}\left(g_{i}\right), i=1, \ldots, k .
$$

By substituting relations (3.5) we get

$$
\left(a_{1}+\sum_{i \geq 3} a_{i} c_{1 i}\right) f_{1} \pm a_{2} f_{2}+\sum_{i \geq 3}\left(a_{i}+a_{2} c_{i 2}\right) f_{i}=0
$$

which gives $a_{2} \in \operatorname{tors}\left(f_{2}\right)=\operatorname{tors}\left(g_{2}\right)$. Therefore, we get

$$
a_{1} g_{1}+\sum_{i=3}^{k} a_{i} g_{i}=0
$$

and consequently

$$
\left(a_{1}+\sum_{i=3}^{k} a_{i} c_{1 i}\right) f_{1}+\sum_{i=3}^{k} a_{i} f_{i}=0
$$

which gives $a_{i} \in \operatorname{tors}\left(f_{i}\right)=\operatorname{tors}\left(g_{i}\right)$ for $i=3, \ldots, k$. So (3.6) reduces to

$$
a_{1} g_{1}=a_{1} f_{1}=0
$$

which gives $a_{1} \in \operatorname{tors}\left(f_{1}\right)=\operatorname{tors}\left(g_{1}\right)$.

By using proposition 3.4 we get:

Theorem 2. Let $\mathcal{S}:=(K, R, w)$ be a weighted sheaf over a simplicial complex $K$. Let $\mathcal{M}$ be an acyclic matching for $\mathcal{S}$. Then one has an isomorphism

$$
H_{*}\left(L_{*}(K), \partial\right) \cong H_{*}\left(C_{*}^{\mathcal{M}}, \partial^{\mathcal{M}}\right)
$$

between the homology of the associated weighted complex $L_{*}(K)$ and the homology of the Morse complex $C_{*}^{\mathcal{M}}$ of $\mathcal{S}$ (definition 3.3).

Proof. The proof is a Gauss-type triangularization of the set of generators of $L_{*}$ (see [19] for free modules).

Let us give an ordering to the pairs in $\mathcal{M}: p_{1}=\left(\tau_{1}, \sigma_{1}\right), p_{2}=\left(\tau_{2}, \sigma_{2}\right), \ldots, p_{m}=$ $\left(\tau_{m}, \sigma_{m}\right)$. Let

$$
\mathcal{M}^{(k)}:=\left\{p_{i} \in \mathcal{M}: i \leq k\right\}
$$

$k=0, \ldots, m$, where we set $\mathcal{M}^{(0)}=\emptyset$ (and we have $\mathcal{M}^{(m)}=\mathcal{M}$ ). Clearly $\mathcal{M}^{(k)}$ is also an acyclic matching for $\mathcal{S}$. Let $C r_{k}$ be the set of critical simplices for $\mathcal{M}^{(k)}$.

Let $\bar{e}_{\sigma}^{(0)}=\bar{e}_{\sigma}$, for all $\sigma \in K$. By induction on $k \geq 0$, assume we have constructed generators

$$
\bar{e}_{\sigma}^{(k)}, \sigma \in K, k \geq 0
$$

for $L_{*}$ such that:

(1) there is a direct sum decomposition into subcomplexes

$$
L_{*}=A_{k} \oplus_{\sigma \in C r_{k}} \frac{R}{\left(w_{\sigma}\right)} \bar{e}_{\sigma}^{(k)},
$$


where $A_{k}$ decomposes as

$$
A_{k}=\oplus_{i=1}^{k} \Gamma_{i}
$$

into elementary acyclic subcomplexes

$$
\Gamma_{i}:=\left[0 \rightarrow \frac{R}{\left(w_{\sigma_{i}}\right)} \bar{e}_{\sigma_{i}}^{(k)} \rightarrow \frac{R}{\left(w_{\tau_{i}}\right)} \bar{e}_{\tau_{i}}^{(k)} \rightarrow 0\right]
$$

(by definition: $w_{\sigma_{i}}=w_{\tau_{i}}$; it follows that $A_{k}$ is acyclic);

(2) the boundary $\partial$ induces on $C r_{k}$ a boundary $\partial^{(k)}$, which is given by formula (3.2) with respect to $\mathcal{M}^{(k)}$.

When $k=0$ we have $A_{0}=0$ and $\partial^{(0)}=\partial$.

Now we consider $\mathcal{M}^{(k+1)}=\mathcal{M}^{(k)} \cup\left\{\left(\tau_{k+1}, \sigma_{k+1}\right)\right\}$. By the acyclicity of $\mathcal{M}^{(k+1)}$, there were no alternating paths in $\mathcal{M}^{(k)}$ between $\sigma_{k+1}$ and $\tau_{k+1}$ except the trivial one $\sigma_{k+1} \triangleright \tau_{k+1}$, so that the coefficient of $\bar{e}_{\tau_{k+1}}^{(k)}$ in $\partial^{(k)} \bar{e}_{\sigma_{k+1}}^{(k)}$ is just the incidence number $\left[\tau_{k+1}: \sigma_{k+1}\right]$. Now we substitute

$$
\bar{e}_{\tau_{k+1}}^{(k+1)}:=\partial^{(k)} \bar{e}_{\sigma_{k+1}}^{(k)}=\left[\tau_{k+1}: \sigma_{k+1}\right] \bar{e}_{\tau_{k+1}}^{(k)}+\sum_{\substack{\tau \in C r_{k} \\|\tau|=|\sigma|-1}} c_{\tau, \sigma_{k+1}} \bar{e}_{\tau}^{(k)},
$$

where the coefficients are here as in (3.2).

Note that $\bar{e}_{\tau_{k+1}}^{(k+1)}$ has the same torsion as $\bar{e}_{\tau_{k+1}}^{(k)}$ (and as $\bar{e}_{\sigma_{k+1}}^{(k)}$ ) by definition of weighted matching and by the fact that going down into the poset gives an element whose weight is a divisor of the weight above.

Now, for all $\sigma$ such that $\sigma \in C r_{k}$ and $\operatorname{dim}(\sigma)=\operatorname{dim}\left(\sigma_{k+1}\right)$ we make the substitution

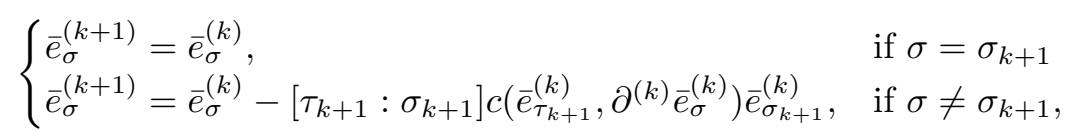

where we set $c\left(\bar{e}_{\tau_{k+1}}^{(k)}, \partial^{(k)} \bar{e}_{\sigma}^{(k)}\right)$ as the coefficient of $\bar{e}_{\tau_{k+1}}^{(k)}$ in $\partial^{(k)} \bar{e}_{\sigma}^{(k)}$ (as given in (3.2)). As before, the torsion of $\bar{e}_{\sigma}^{(k+1)}$ equals that of $\bar{e}_{\sigma}^{(k)}$.

For the remaining elements, we set

$$
\bar{e}_{\sigma}^{(k+1)}=\bar{e}_{\sigma}^{(k)} .
$$

By applying proposition (3.4) with

$$
f_{1}=\bar{e}_{\sigma_{k+1}}^{(k)}, \quad f_{2}:=\bar{e}_{\tau_{k+1}}^{(k)},
$$

and enumerating $f_{i}, i \geq 3$, subsequently all other $\bar{e}_{\sigma}^{(k)} \in C r_{k} \backslash\left(\sigma_{k+1} \cup \tau_{k+1}\right)$, and setting, respectively

$$
g_{1}=f_{1}=\bar{e}_{\sigma_{k+1}}^{(k+1)}, \quad g_{2}=\partial^{(k)} \bar{e}_{\sigma_{k+1}}^{(k+1)}=\bar{e}_{\tau_{k+1}}^{(k+1)}
$$

and all others $g_{i}$ as in (3.8), we get

$$
L_{*}=A_{k+1} \oplus_{\sigma \in C r_{k+1}} \frac{R}{\left(w_{\sigma}\right)} \bar{e}_{\sigma}^{(k+1)},
$$

where

$$
A_{k+1}=A_{k} \oplus \Gamma_{k+1},
$$


with

$$
\Gamma_{k+1}:=\left[0 \rightarrow \frac{R}{\left(w_{\sigma_{k+1}}\right)} \bar{e}_{\sigma_{k+1}}^{(k+1)} \rightarrow \frac{R}{\left(w_{\tau_{k+1}}\right)} \bar{e}_{\tau_{k+1}}^{(k+1)} \rightarrow 0\right]
$$

By (3.8) the boundary changes only for the alternating paths containing the edge $\left(\bar{e}_{\sigma_{k+1}}, \bar{e}_{\tau_{k+1}}\right)$, so we get formula $(3.2)$.

Let us consider the filtration of the Morse complex into increasing weights:

$$
F^{p} C_{*}^{\mathcal{M}}:=\bigoplus_{\substack{\sigma \text { critical } \\ v_{\varphi}(\sigma) \leq p}} \frac{R}{\left(\varphi^{v_{\varphi}(\sigma)}\right)} \bar{e}_{\sigma}
$$

and let

$$
\mathcal{F}^{p} C_{*}^{\mathcal{M}}:=F^{p} C_{*}^{\mathcal{M}} / F^{p-1} C_{*}^{\mathcal{M}}
$$

be the quotient complex. As an $R$-module we have

$$
\mathcal{F}^{p} C_{*}^{\mathcal{M}} \cong \bigoplus_{\substack{\sigma \text { critical } \\ v_{\varphi}(\sigma)=p}} \frac{R}{\left(\varphi^{p}\right)} \bar{e}_{\sigma}
$$

Then as a corollary of theorem 2 we can reformulate theorem 1 in terms of the Morse complex as follows.

Theorem 3. Let $\mathcal{S}$ and $\varphi$ be as above and let $\mathcal{M}$ be an acyclic matching for $\mathcal{S}_{(\varphi)}$. Then the spectral sequence of theorem 1 is computed by

$$
E_{p, q}^{1} \cong H_{p+q}\left(\mathcal{F}^{p} C_{*}^{\mathcal{M}}\right)
$$

where $C_{*}^{\mathcal{M}}$ is the Morse complex of $\mathcal{S}_{(\varphi)}$.

Proof. We note that, as in the proof of theorem 2, $L_{(\varphi)}$ is the direct sum $A_{m} \oplus C_{*}$ of an acyclic complex and of the Morse complex. This splitting is compatible with the filtrations, therefore the theorem follows straightforward.

\section{Artin groups}

We consider here finitely generated Coxeter systems $(\mathbf{W}, S)$, (i.e., $S$ is finite). Recall that

$$
\mathbf{W}=\left\langle s \in S \mid\left(s s^{\prime}\right)^{m\left(s, s^{\prime}\right)}=1\right\rangle,
$$

where $m\left(s, s^{\prime}\right) \in \mathbb{N} \cup\{\infty\}, m\left(s, s^{\prime}\right)=m\left(s^{\prime}, s\right), m(s, s)=1([2,17,25])$. We recall some general facts.

The group $\mathbf{W}$ can be realized as a group generated by (in general, non-orthogonal) reflections in $\mathbb{R}^{n}, n=|S|$. Let $\mathcal{A}$ be the reflection arrangement, i.e.,

$$
\mathcal{A}=\left\{H \subset \mathbb{R}^{n} \mid H \text { is fixed by some reflection in } \mathbf{W}\right\} .
$$

We consider the stratification into facets of $\mathbb{R}^{n}$ induced by $\mathcal{A}$, where the co-dimension0 facets, (i.e., the connected components of the complement to the arrangement) are called chambers. Fix a base-chamber $C_{0}$. The Coxeter generator set $S$ corresponds to the set of reflections with respect to the walls of $C_{0}$.

Let $U:=\mathbf{W} \cdot \bar{C}_{0}$ be the orbit of the closure of the base chamber. $U$ is called the Tits cone of the Coxeter system. 
Each chamber contained inside $U$ is of the shape $w \cdot C_{0}$ for a unique $w \in \mathbf{W}$. Of course, $\mathcal{A}$ is not locally finite if $\mathbf{W}$ is infinite (e.g., 0 is contained in all the hyperplanes). The orbits of the facets of $C_{0}$ give a "stratification" of $U$ into relatively open cells, also called facets (in general, $U$ is neither open nor closed in $\mathbb{R}^{n}$ ).

Recall also:

(1) $U$ is a convex cone in $\mathbb{R}^{n}$ with vertex 0 .

(2) $U=\mathbb{R}^{n}$ iff $\mathbf{W}$ is finite

(3) The stabilizer of a facet $F$ in $U$ is the subgroup $\mathbf{W}_{F}$ generated by all the reflections with respect to hyperplanes (in $\mathcal{A}$ ) containing $F$. So, in general $\mathbf{W}_{F}$ is not finite.

(4) $U^{0}:=\operatorname{int}(U)$ is open in $\mathbb{R}^{n}$ and a (relatively open) facet $F \subset \bar{C}_{0}$ is contained in $U^{0}$ iff the stabilizer $\mathbf{W}_{F}$ is finite.

By property 4 the arrangement is locally finite in the interior part $U^{0}$.

Let $H_{\mathbb{C}}:=H+i H \subset \mathbb{C}^{n}$ be the complexification of the hyperplane $H$, and set

$$
\mathbf{Y}:=\left[U^{0}+i \mathbb{R}^{n}\right] \backslash \cup_{H \in \mathcal{A}} H_{\mathbb{C}}
$$

which corresponds to complexifying only the interior part of the Tits cone. The group $\mathbf{W}$ acts (as before) diagonally over $\mathbf{Y}$, and one shows easily (by property 3 above)l that the action is free. Therefore, one has an orbit space

$$
\mathbf{Y}_{\mathbf{W}}:=\mathbf{Y} / \mathbf{W}
$$

which is still a manifold.

Definition 4.1. Define

$$
\mathbf{G}_{\mathbf{W}}:=\pi_{1}\left(\mathbf{Y}_{\mathbf{W}}\right)
$$

as the Artin group of type $\mathbf{W}$.

(see $[3,13]$ for the case when $\mathbf{W}$ is finite). It is known that the orbit space $\mathbf{Y}_{\mathbf{W}}$ deformation retracts onto a $C W$-complex $\mathbf{X}_{\mathbf{W}}$, which is explicitly described in combinatorial terms (see [23] and also [12,22], and the recent paper [21], where also a survey of the theory is given). From the explicit description of $\mathbf{X}_{\mathbf{W}}$ one derives a presentation of $\mathbf{G}_{\mathbf{W}}$

$$
\mathbf{G}_{\mathbf{W}}=\left\langle g_{s}, s \in S \mid g_{s} g_{s^{\prime}} g_{s} \cdots=g_{s^{\prime}} g_{s} g_{s^{\prime}} \ldots\right\rangle
$$

(same number $m\left(s, s^{\prime}\right)$ of factors on each side, where we have to consider only pairs such that $m\left(s, s^{\prime}\right)$ is finite) as well as an algebraic complex, which computes the homology (and cohomology) of any local system over the orbit space $\mathbf{Y}_{\mathbf{W}}$. When such space is of type $K(\pi, 1)$ this cohomology equals that of the group $\mathbf{G}_{\mathbf{W}}$.

We recall formulas in case of the following representation (one can see that such representation corresponds, for the case of the braid group, to the determinant of the Burau representation; in general, there is a precise relation with the (co)-homology of the Milnor fiber of a suitable fibration; see, for example, $[4,9])$.

Let $R:=A\left[q, q^{-1}\right]$ be the ring of Laurent polynomials over a ring $A$. One can represent $\mathbf{G}_{\mathbf{W}}$ by

$$
g_{s} \mapsto[\text { multiplication by }-q], \quad \forall s \in S
$$

$(\in \operatorname{Aut}(R))$. 
First we denote by $K:=K(\mathbf{W})$ the simplicial scheme, defined over $S$, of the subsets $J \subset S$, which generate a finite parabolic subgroup $\mathbf{W}_{J}$ (we include the empty set which by definition generates the trivial subgroup). The algebraic complex, which computes the trivial simplicial homology of $K$ with coefficients in $\mathbb{Z}$ is given in (2.2) and (2.3).

Notice that $C_{*}^{0}$ is graduated according to the cardinality of the subsets, so there is a degree-1 shift isomorphism with the standard complex for the simplicial homology of $K$, i.e., $H_{m}\left(C_{*}^{0}\right) \cong H_{m-1}(K)$ for $m \geq 1$.

The complex which computes the homology of $\mathbf{Y}_{\mathbf{W}}$ is given by

$$
C_{k}:=\bigoplus_{\substack{J \subset S \\|J|=k \\ \mathbf{w}_{J} \text { finite }}} R \cdot e_{J}
$$

with boundary

$$
\partial\left(e_{J}\right)=\sum_{\substack{I \subset J \\|I|=|J|-1,}}[I: J] \frac{\mathbf{W}_{J}(q)}{\mathbf{W}_{I}(q)} e_{I}
$$

where

$$
\mathbf{W}_{J}(q):=\sum_{w \in W_{J}} q^{\ell(w)}
$$

is the Poincaré series of the group $\mathbf{W}_{J}$ (here, a polynomial since $\mathbf{W}_{J}$ is finite by definition). The denominator $\mathbf{W}_{I}(q)$ divides the numerator $\mathbf{W}_{J}(q)$ so the quotient is still a polynomial.

We can formally rewrite the boundary map in (4.5) as:

$$
\partial\left(\frac{1}{\mathbf{W}_{J}(q)} e_{J}\right)=\sum_{\substack{I \subset J \\|J|=|I|+1}}[I: J] \frac{1}{\mathbf{W}_{I}(q)} \cdot e_{I}
$$

That means that the fractions $e_{J} / \mathbf{W}_{J}(q)$ behave like the cells of the simplicial scheme $K$.

Consider the diagonal map:

$$
\Delta: C_{*} \rightarrow C_{*}^{0}, \quad e_{J} \mapsto \mathbf{W}_{J}(q) e_{J}^{0} .
$$

Clearly $\Delta$ is an injective chain-complex homomorphism, so there is an exact sequence of complexes:

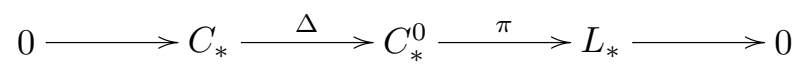

where

$$
L_{k}:=\bigoplus_{\substack{J \subseteq S \\ J|=k\\| \mathbf{W}_{J} \mid<\infty}} \frac{R}{\left(\mathbf{W}_{J}(q)\right)} \cdot \bar{e}_{J}
$$

is the quotient complex.

Passing to the associated long exact sequence we get:

$$
\stackrel{\pi_{*}}{\longrightarrow} H_{k+1}\left(L_{*}\right) \stackrel{\delta}{\longrightarrow} H_{k}\left(C_{*}\right) \stackrel{\Delta_{*}}{\rightarrow} H_{k}\left(C_{*}^{0}\right) \stackrel{\pi_{*}}{\rightarrow} H_{k}\left(L_{*}\right) \stackrel{\delta}{\longrightarrow} H_{k-1}\left(C_{*}\right) \stackrel{\Delta_{*}}{\longrightarrow}
$$


Remark 4.2. With the preceding notations, we can consider the map $w: K \rightarrow R$ given by

$$
J \longrightarrow w_{J}:=\mathbf{W}_{J}(q) \in R \text {. }
$$

Since $w_{I} \mid w_{J}$ if $I \subset J$ we can see the $w_{J}$ 's as weights associated to the simplices of the simplicial complex $K$. It is clear that

$$
(K, R, w)
$$

is a weighted sheaf over $K$, and that $L_{*}$ is the associated weighted complex.

\section{Matchings for the affine groups}

From the fundamental Remark 4.2, and from sequences (4.8) and (4.9), we can compute the local homology of the Artin group from the knowledge of the associated weighted complex $L_{*}$ (we assume $A$ is a field so $R=A\left[q^{ \pm 1}\right]$ is a PID).

In this section, we show how to find good matchings for the affine-type Artin groups, so that we can fruitfully use the theory developed in Section 3.

We make use of theorems 1 and 3. In particular, we need a weighted matching which allows us to reduce computations to small Morse complexes.

Recall that a standard way to produce acyclic matchings is given by the following easy lemma (see [18, Lemma 4.2], [19, Theorem 11.10])

Lemma 5.1. Let $f: \mathcal{P} \rightarrow \mathcal{Q}$ be a poset map and for any $q \in Q$ let $\mathcal{M}_{q}$ be an acyclic matching on $f^{-1}(q)$. Then

is an acyclic matching on $\mathcal{P}$.

$$
\mathcal{M}:=\cup_{q \in Q} \mathcal{M}_{q}
$$

In case of an affine Artin group $\mathbf{G}_{\mathbf{W}}$, associated to a Coxeter system $(\mathbf{W}, S)$, notice that the associated simplicial scheme $K=K(\mathbf{W})$, defined in part 4, is given by the scheme of all proper subsets of $S$.

Let us give any total ordering $<$ to $S:=\left\{s_{1}<s_{2}<\cdots<s_{n}\right\}$. We have

Lemma 5.2. The map $f: K \rightarrow S$ defined by

$$
K \ni J \longrightarrow f(J):=\min \{s \in S: s \notin J\}
$$

is well defined and it is a poset map.

Proof. The map $f$ is well defined because each $J \in K$ misses some element from $S$.

If $I \subset J$ then clearly $f(I) \leq f(J)$, so $f$ is a poset map.

Remark 5.3. Let

$$
K_{i}:=\left\{J \subset K: s_{i} \notin J\right\} .
$$

Clearly $K_{i}$ is the full simplex of all the subsets of $S \backslash\left\{s_{i}\right\}$ (including the empty set). So one has an isomorphism

$$
f^{-1}\left(s_{i}\right) \cong S t_{K_{i}}\left(\left\{s_{1}, \ldots s_{i-1}\right\}\right), \quad i \geq 0 .
$$

Note that all the $f^{-1}\left(s_{i}\right)$ correspond to the case where one vertex is removed from the Coxeter diagram, so reducing to a finite-type Artin group. Starting from exceptional affine cases, one will have exceptional finite-type Artin groups, which were worked out in [9] by different methods. 


\section{Computational results}

The computations of the $E_{1}$-page of the spectral sequence introduced in Section 2 use a weighted matching on $L_{*}$ of the type described in the previous section, together with theorems 1 and 3 . As a matter of fact, in all cases that we consider we succeed to get matchings such that the Morse complex is minimal at each level $p$, meaning that the boundary of the Morse complex of $F^{p} L_{(\varphi)} / F^{p-1} L_{(\varphi)}$ vanishes.

One can work out the differential $d_{1}$ in the spectral sequence introduced before as follows.

Theorem 4. Let $\mathcal{S}, \varphi$, be as before. The differential

$$
d_{p, q}^{1}: E_{p, q}^{1} \rightarrow E_{p-1, q}^{1}
$$

is induced by the boundary of the Morse complex

$$
H_{p+q}\left(\mathcal{F}^{p} C_{*}^{\mathcal{M}}\right) \rightarrow H_{p+q-1}\left(\mathcal{F}^{p-1} C_{*}^{\mathcal{M}}\right)
$$

The boundary of a critical class $\left[\bar{e}_{J}\right] \in H_{p+q}\left(F^{p} / F^{p-1}\right)$ is computed as in formula (3.2), through all alternating paths $p$ starting from $\bar{e}_{J}$.

Proof. In fact, the decomposition of $L_{(\varphi)}$ in $A_{m} \oplus C_{*}^{\mathcal{M}} f$ obtained in the proof of theorem 2, where $A_{m}$ is an acyclic subcomplex while $C_{*}^{\mathcal{M}}$ is the Morse complex, clearly induces an isomorphism of spectral sequences

$$
E_{p, q}^{*}\left(L_{(\varphi)}\right) \cong E_{p, q}^{*}\left(C_{*}^{\mathcal{M}}\right)
$$

Then we use that the Morse complex is minimal at each level $p$ (see above) to identify homology classes with classes of the shape $\left[\bar{e}_{J}\right]$.

To explain the procedure, we give in detail the example of $\widetilde{\mathrm{F}}_{4}$, in the case of $\varphi_{2^{-}}$ primary component. The Coxeter diagram is given here by:

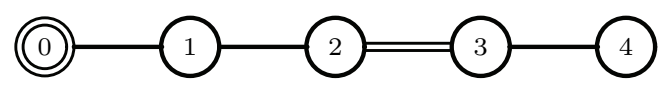

The weighted sheaf $\left(K, R, w_{\left(\varphi_{2}\right)}\right)$ corresponding to the $\varphi_{2}$-primary component is associated to the weighted complex $L_{\left(\varphi_{2}\right)}:=L_{\left(\varphi_{2}\right)}\left(\widetilde{\mathrm{F}}_{4}\right)$.

We have the following weighted acyclic matching over $L_{\left(\varphi_{2}\right)}$. We give the Hasse diagram, where the pairs of the matching are joined by bold lines. 


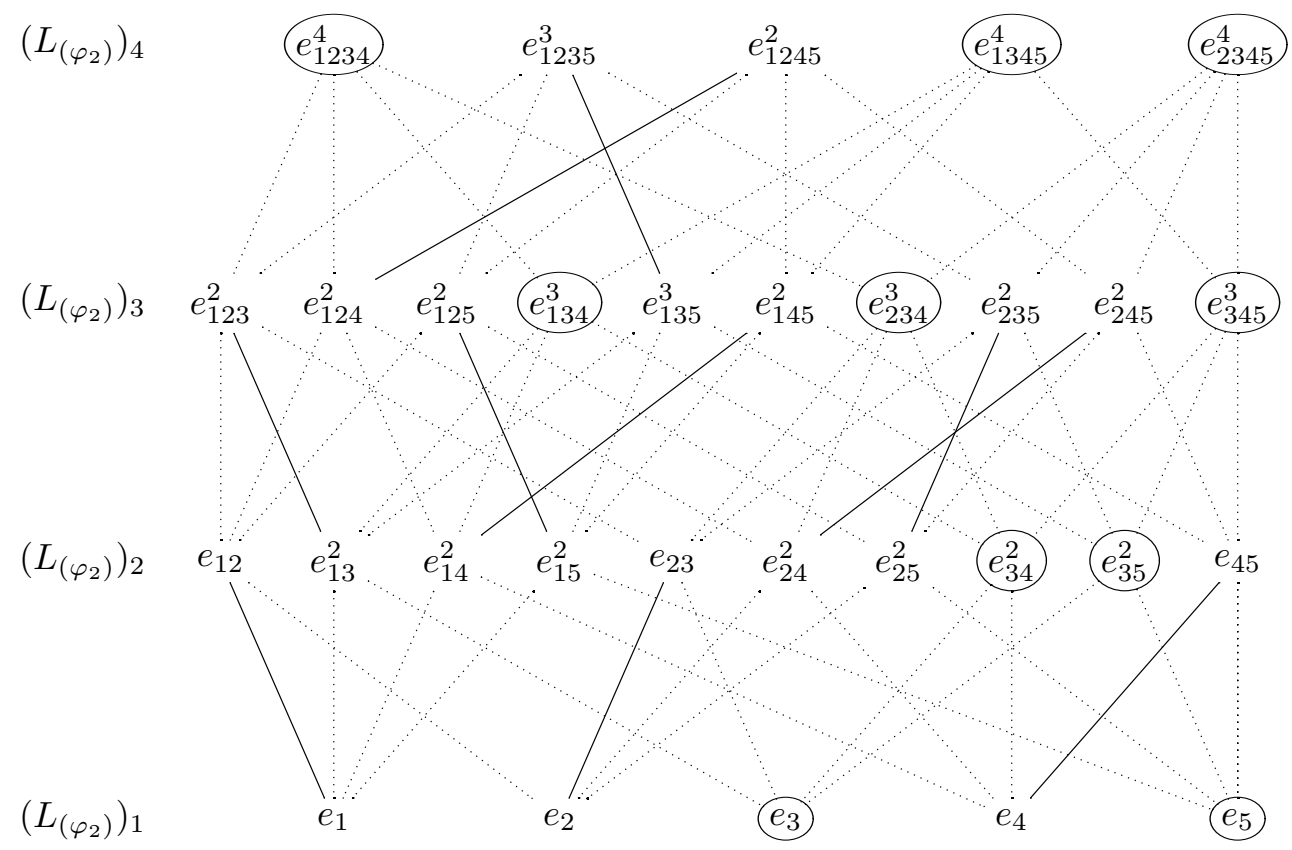

In view of theorem 3 , we have that the Morse complex $\mathcal{C}_{*}^{\mathcal{M}}$ associated to the given matching is generated by the critical elements, which are circled in the diagram.

Note that the boundaries in $\left(F^{i} / F^{i-1}\right)_{*}$ over the critical cells are vanishing. This allows us to use the same notations for the classes generating the homology groups. We obtain the first page of the spectral sequence. The only non-zero entries are (we drop the index $\varphi_{2}$ for brevity):

$$
\begin{aligned}
& E_{1,0}^{1}:=H_{1}\left(\mathcal{F}^{1} C_{*}^{\mathcal{M}}\right) \cong H_{1}\left(F^{1} / F^{0}\right)=\frac{R}{\left(\varphi_{2}\right)} e_{3} \oplus \frac{R}{\left(\varphi_{2}\right)} e_{5}, \\
& E_{2,0}^{1}:=H_{2}\left(\mathcal{F}^{2} C_{*}^{\mathcal{M}}\right)=H_{2}\left(F^{2} / F^{1}\right)=\frac{R}{\left(\varphi_{2}^{2}\right)} e_{34} \oplus \frac{R}{\left(\varphi_{2}^{2}\right)} e_{35}, \\
& E_{3,0}^{1}:=H_{3}\left(\mathcal{F}^{3} C_{*}^{\mathcal{M}}\right)=H_{3}\left(F^{3} / F^{2}\right)=\frac{R}{\left(\varphi_{2}^{3}\right)} e_{134} \oplus \frac{R}{\left(\varphi_{2}^{3}\right)} e_{234} \oplus \frac{R}{\left(\varphi_{2}^{3}\right)} e_{345}, \\
& E_{4,0}^{1}:=H_{4}\left(\mathcal{F}^{4} C_{*}^{\mathcal{M}}\right)=H_{4}\left(F^{4} / F^{3}\right)=\frac{R}{\left(\varphi_{2}^{4}\right)} e_{1234} \oplus \frac{R}{\left(\varphi_{2}^{4}\right)} e_{1345} \oplus \frac{R}{\left(\varphi_{2}^{4}\right)} e_{2345} .
\end{aligned}
$$

Furthermore, we have only three non-zero differentials, which are calculated using formula (3.2):

1. $d_{2,0}^{1}: E_{2,0}^{1} \rightarrow E_{1,0}^{1}$.

The alternating paths starting from $e_{34}$ and ending in $e_{3}$ or in $e_{5}$ are unique:

$$
e_{34} \triangleright e_{3} \quad e_{34} \triangleright e_{4} \unlhd e_{45} \triangleright e_{5}
$$


TABLE 1. $z$ as defined at the end

\begin{tabular}{ccccc}
\hline & $\widetilde{A}_{1}$ & $\widetilde{B}_{2}$ & $\widetilde{I}_{2}(6)$ & $\widetilde{F}_{4}$ \\
\hline$H_{0}$ & $\frac{R}{\left(\varphi_{2}\right)} \frac{\partial\left(e_{1}\right)}{\left(\varphi_{2}\right)}$ & $\frac{R}{\left(\varphi_{2}\right)} \frac{\partial\left(e_{1}\right)}{\left(\varphi_{2}\right)}$ & $\frac{R}{\left(\varphi_{2}\right)} \frac{\partial\left(e_{1}\right)}{\left(\varphi_{2}\right)}$ & $\frac{R}{\left(\varphi_{2}\right)} \frac{\partial\left(e_{1}\right)}{\left(\varphi_{2}\right)}$ \\
$H_{1}$ & $R z$ & $\frac{R}{\left(\varphi_{2} \varphi_{4}\right)} \frac{\partial\left(e_{12}\right)}{\left(\varphi_{2} \varphi_{4}\right)}$ & $\frac{R}{\left(\varphi_{2} \varphi_{3}\right)} \frac{\partial\left(e_{23}\right)}{\left(\varphi_{2} \varphi_{3}\right)}$ & $\frac{R}{\left(\varphi_{2}\right)} \frac{\partial\left(e_{34}\right)}{\left(\varphi_{2}\right)}$ \\
$H_{2}$ & 0 & $R \frac{R\left(e_{13}\right)}{\left(\varphi_{2}\right)}$ & $R z$ & $\frac{R}{\left(\varphi_{2} \varphi_{3}\right)} \frac{\partial\left(e_{234}\right)}{\varphi_{2} \varphi_{3}}$ \\
$H_{3}$ & 0 & 0 & 0 & $R$ \\
$H_{4}$ & 0 & 0 & 0 & $\frac{\partial\left(e_{2345}\right)}{\left(\varphi_{2} \varphi_{3} \varphi_{4} \varphi_{8}\right)}$ \\
\hline$\left.\varphi_{2} \varphi_{3} \varphi_{4} \varphi_{8}\right)$ & $\frac{\partial\left(e_{2345}\right)}{\left(\varphi_{2}\right)}$
\end{tabular}

So $d_{2,0}^{1}\left(e_{34}\right)=e_{3}-e_{5}$. The same if we consider alternating paths between $e_{35}$ and $e_{3}$ or $e_{5}$ :

$$
e_{35} \triangleright e_{3} \quad e_{35} \triangleright e_{5} .
$$

In this case, we obtain $d_{2,0}^{1}\left(e_{35}\right)=e_{3}-e_{5}$.

2. $d_{3,0}^{1}: E_{3,0}^{1} \rightarrow E_{2,0}^{1}$.

The alternating paths between critical cells are:

$$
\begin{array}{cc}
e_{134} \triangleright e_{34} & e_{134} \triangleright e_{14} \unlhd e_{145} \triangleright e_{15} \unlhd e_{125} \triangleright e_{25} \unlhd e_{235} \triangleright e_{35} \\
e_{234} \triangleright e_{34} & e_{234} \triangleright e_{24} \unlhd e_{245} \triangleright e_{25} \unlhd e_{235} \triangleright e_{35} \\
e_{345} \triangleright e_{34} & e_{345} \triangleright e_{35}
\end{array}
$$

They determine the differential $d_{3,0}^{1}$ :

$d_{3,0}^{1}\left(e_{134}\right)=d_{3,0}^{1}\left(e_{234}\right)=d_{3,0}^{1}\left(e_{345}\right)=-e_{34}+e_{35}$.

3. $d_{4,0}^{1}: E_{4,0}^{1} \rightarrow E_{3,0}^{1}$.

Finally, the alternating paths between critical cells are:

$e_{1234} \triangleright e_{134} \quad e_{1234} \triangleright e_{234} \quad e_{1345} \triangleright e_{134} \quad e_{1345} \triangleright e_{345} \quad e_{2345} \triangleright e_{234} \quad e_{2345} \triangleright e_{345}$

And the differential $d_{4,0}^{1}$ is given by:

$$
\begin{aligned}
& d_{4,0}^{1}\left(e_{1234}\right)=-e_{234}+e_{134}, \\
& d_{4,0}^{1}\left(e_{1345}\right)=-e_{345}+e_{134}, \\
& d_{4,0}^{1}\left(e_{2345}\right)=-e_{345}+e_{234} .
\end{aligned}
$$




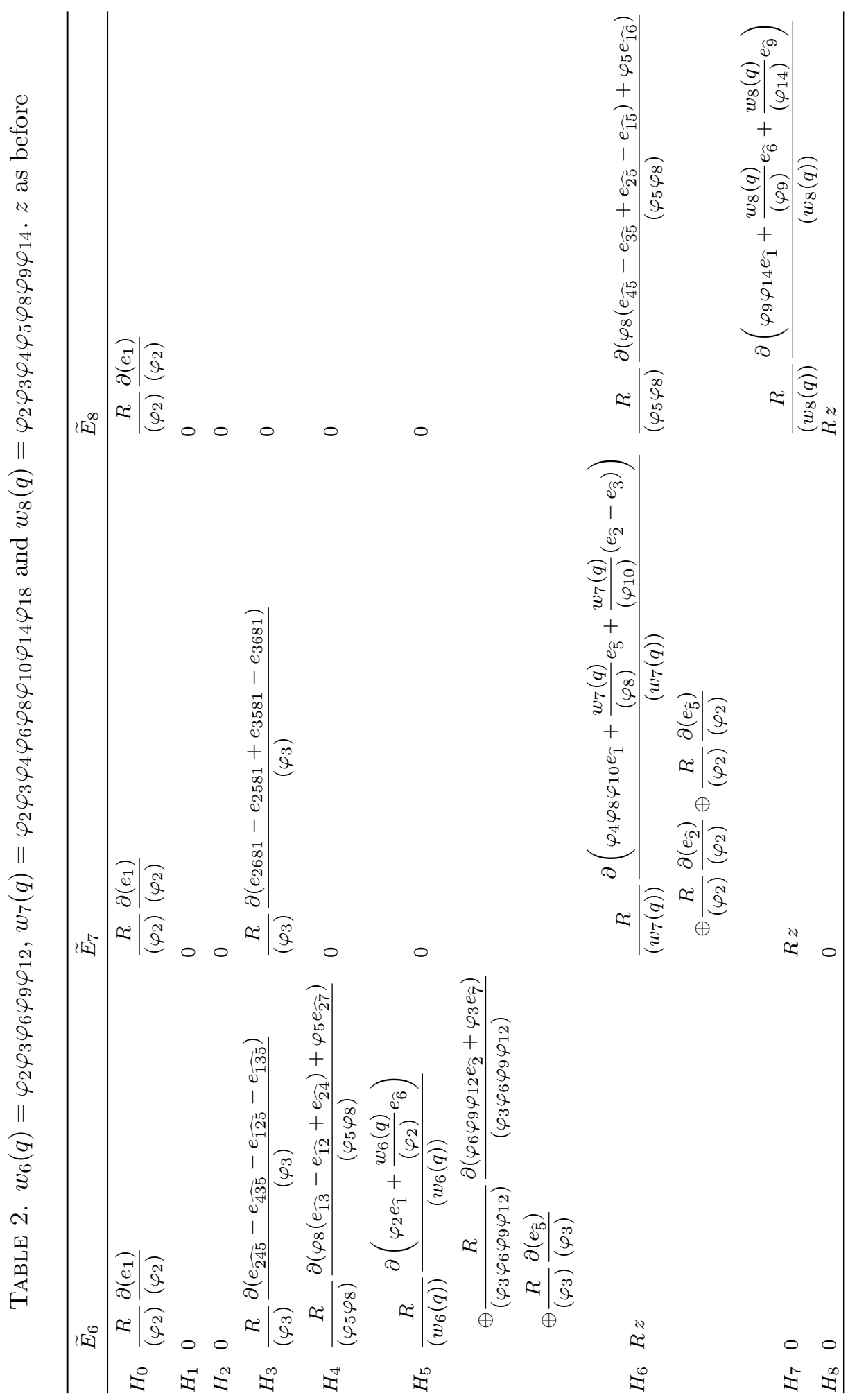


We obtain the homology groups for the complex $\left(L_{\left(\varphi_{2}\right)}\right)_{*}$ :

$$
\begin{aligned}
H_{4}\left(L_{\left(\varphi_{2}\right)}\right)= & \frac{R}{\left(\varphi_{2}^{4}\right)}\left(e_{1234}-e_{1235}+e_{1245}-e_{1345}+e_{2345}\right) \\
& \oplus \frac{R}{\left(\varphi_{2}\right)}\left(\varphi_{2}^{3} e_{1234}\right) \oplus \frac{R}{\left(\varphi_{2}\right)}\left(\varphi_{2}^{3} e_{2345}\right), \\
H_{3}\left(L_{\left(\varphi_{2}\right)}\right)= & \frac{R}{\left(\varphi_{2}\right)}\left(\varphi_{2}^{2} e_{234}\right), \\
H_{2}\left(L_{\left(\varphi_{2}\right)}\right)= & \frac{R}{\left(\varphi_{2}\right)}\left(\varphi_{2} e_{34}\right), \\
H_{1}\left(L_{\left(\varphi_{2}\right)}\right)= & \frac{R}{\left(\varphi_{2}\right)}\left(e_{3}\right) .
\end{aligned}
$$

Similar computation is done for the $\varphi_{d}$ - torsion, $d \geq 3$.

We collect in Tables 1 and 2 the final results of our computations. We use the computation of the homology of the weighted complex $L_{*}$ together with the exact sequences (4.8) and (4.9).

As remarked in Section 5 the simplicial complex of subsets generating finite parabolic subgroups is given by all the proper subsets of $S$, so it is the boundary of a simplex, having reduced homology only in the top dimension. By this remark and the exact sequence (4.9) it is easy to pass from the homology of the weighted complex to that of the Artin group.

The entries of the tables give the module $H_{*}$ of the corresponding group, together with representative generators.

For an affine Dynkin graph $\Gamma$ with $n+1$ vertices, we define $\mathbf{W}_{n}(q)$ to be the lcm between the Poincaré polynomials ranging over the subgraphs with exactly $n$ vertices. We denote by (see formula 4.5) $z=\sum_{i \in I}[\Gamma \backslash\{i\}: \Gamma] \frac{\mathbf{W}_{n}(q)}{\mathbf{W}_{\Gamma \backslash\{i\}}(q)} e_{\Gamma \backslash\{i\}}$ the weighted alternating sum.

\section{References}

[1] K. Baclawski, Whitney numbers of geometric lattices, Adv. Math. 16 (1975), 125-138.

[2] N. Bourbaki, Groupes et Algebrès de Lie, vol. Chapters IV-VI, Hermann, 1968.

[3] E. Brieskorn, Die Fundamentalgruppe des Raumes der regulären orbits einer endlichen komplexen Spiegelunsgruppe, Invent. Math. 12 (1971), 57-61.

[4] F. Callegaro, The homology of the Milnor fiber for classical braid groups, 2005, arXiv:math.AT/0511453.

[5] - The homology of the Milnor fiber for classical braid groups, Algebr. Geom. Topol. 6 (2006), 1903-1923.

[6] F. Callegaro, D. Moroni and M. Salvetti, Cohomology of affine Artin groups and applications, Trans. Amer. Math. Soc. 360 (2008), 4169-4188.

[7] - Cohomology of Artin groups of type $\tilde{A}_{n}, \tilde{B}_{n}$ and applications, Geom. Topol. Monogr. 13 (2008), 85-104.

[8] - The $K(\pi, 1)$ problem for the affine Artin group of type $\tilde{B}_{n}$ and its cohomology, J. Eur. Math. Soc. 12 (2010), 1-22.

[9] F. Callegaro and M. Salvetti, Integral cohomology of the Milnor fibre of the discriminant bundle associated with a finite Coxeter group, C. R. Acad. Sci. Paris, Ser. I 339 (2004), 573-578.

[10] C. De Concini, C. Procesi and M. Salvetti, Arithmetic properties of the cohomology of braid groups, Topology 40(4) (2001), 739-751. 
[11] C. De Concini, C. Procesi, M. Salvetti and F. Stumbo, Arithmetic properties of the cohomology of Artin groups, Ann. Sci. Norm. Super. Pisa Cl. Sci. 28(4) (1999), 695-717.

[12] C. De Concini and M. Salvetti, Cohomology of Artin groups, Math. Res. Lett. 3 (1996), 293-297.

[13] P. Deligne, Les immeubles des groupes de tresses généralisés, Invent. Math. 17 (1972), 273-302.

[14] R. Forman, Combinatorial Morse theory on CW-complexes, Adv. Math. 87 (1996), 189-236.

[15] — A user's guide to discrete Morse theory, Sém. Lothar. Combin. 48 (2002), 1-35.

[16] E. V. Frenkel, Cohomology of the commutator subgroup of the braids group, Funct. Anal. Appl. $\mathbf{2 2}(3)$ (1988), 248-250.

[17] J. Humphreys, Reflection groups and Coxeter groups, Cambridge University Press, Cambridge, UK, 1990.

[18] J. Jonnson, "Simplicial Complexes of Graphs" PhD thesis, KTH, Stockholm, 2005.

[19] D. Kozlov, Combinatorial algebraic topology, Springer-Verlag, Berlin, 2008.

[20] D. Moroni, Finite and infinite type Artin groups: topological aspects and cohomological computations, PhD thesis, 2006.

[21] D. Moroni, M. Salvetti and A. Villa, Some topological problems on the configuration spaces of Artin and Coxeter groups, in 'CRM series' in 'Proceedings Configuration Spaces (Geometry, Combinatorics and Topology)', (A. Bjorner, F. Cohen, C. De Concini, C. Procesi and M. Salvetti, eds.), 14 Edizioni della Scuola Normale Superiore, 2012.

[22] M. Salvetti, Topology of the complement of real hyperplanes in $\mathbb{C}^{N}$, Invent. Math. 88(3) (1987), 603-618.

[23] — The Homotopy type of Artin groups, Math. Res. Lett. 1 (1994), 567-577.

[24] - On the cohomology and topology of Artin and Coxeter groups, Pubblicazioni Dipartimento di Matematica L.Tonelli, Pisa, 2005.

[25] E. Vinberg, Discrete linear groups generated by reflections, Math. USSR Izvestija 5(5) (1971), 1083-1119.

[26] S. Yuzvinsky, Cohen-Macaulay rings of sections, Adv. Math. 63 (1987), 172-195.

Dipartimento di Matematica, Università di Pisa, Largo B. Pontecorvo, 5, 56127 Pisa, ITALY

E-mail address: salvetti@dm.unipi.it

Department of Mathematical Sciences, ISI Foundation, Via Alassio 11/C 10126 Torino

- ITALY

E-mail address: avilla@mail.dm.unipi.it 
